Bull. Austral. Math. Soc.

VoL. 57 (1998) [393-401]

\title{
CURVES WITH CONTINUOUS CURVATURES IN EUCLIDEAN SPACES
}

\author{
VITALY USHAKOV
}

A class of curves of minimal smoothness for which one can build the Frenet frame, Frenet's formulae and osculating planes is described.

\section{SMOOTHNESS}

To construct the classical Frenet frame and Frenet equations for a curve $\gamma \subset E^{n}$, one needs the curve to be $C^{n}$ smooth. Indeed, given $\gamma$ in natural (arc-length) parametrisation $r(s)$ (throughout the paper $s$ denotes the natural parameter of a curve), the Frenet frame $\left\{e_{i}(s)\right\}_{1}^{n}$ and the curvature functions $\left\{k_{i}(s)\right\}_{1}^{n-1}$ satisfy the Frenet equations

$$
\left\{\begin{aligned}
\dot{e}_{1} & =k_{1} e_{2} \\
\dot{e}_{2} & =-k_{1} e_{1}+k_{2} e_{3} \\
& \vdots \\
\dot{e}_{i} & =-k_{i-1} e_{i-1}+k_{i} e_{i+1} \\
\vdots & \\
\dot{e}_{n-1} & =-k_{n-2} e_{n-2}+k_{n-1} e_{n} \\
\dot{e}_{n} & =-k_{n-1} e_{n-1}
\end{aligned}\right.
$$

with $e_{1}=\dot{r}$ (see for example, $\left[2\right.$, pp.61-63]). Hence $\left\{e_{i}(s)\right\}_{1}^{n}$ and $\left\{k_{i}(s)\right\}_{1}^{n-1}$ can be

Received 3rd September, 1997

I would like to thank Professor H Rubinstein and Professor W Neumann who kindly afforded me an opportunity to work at the University of Melbourne.

Copyright Clearance Centre, Inc. Serial-fee code: 0004-9729/98 \$A2.00+0.00. 
evaluated from $r(s)$ by the formulae:

$$
\begin{aligned}
k_{1} & =\left\|\dot{e}_{1}\right\| & e_{1} & =\dot{r} \\
k_{2} & =\left\|\dot{e}_{2}+k_{1} e_{1}\right\| & e_{2} & =\frac{1}{k_{1}} \dot{e}_{1} \\
k_{3} & =\left\|\dot{e}_{3}+k_{2} e_{2}\right\| & e_{3} & =\frac{1}{k_{2}}\left(\dot{e}_{2}+k_{1} e_{1}\right) \\
& \vdots & e_{4} & =\frac{1}{k_{3}}\left(\dot{e}_{3}+k_{2} e_{2}\right) \\
k_{n-2} & =\left\|\dot{e}_{n-2}+k_{n-3} e_{n-3}\right\| & e_{n-1} & =\frac{1}{k_{n-2}}\left(\dot{e}_{n-2}+k_{n-3} e_{n-3}\right) \\
k_{n-1} & =\left\|\dot{e}_{n-1}+k_{n-2} e_{n-2}\right\| & e_{n} & =\frac{1}{k_{n-1}}\left(\dot{e}_{n-1}+k_{n-2} e_{n-2}\right) .
\end{aligned}
$$

Naturally, we assume that the curve is essentially $n$-dimensional, that is, all the curvatures are positive. Therefore, the radius vector $r(s)$ has to be differentiated $n$ times and it seems reasonable to require the curve to be $C^{n}$ smooth. In such a case we have

$$
\begin{aligned}
& k_{1} \in C^{n-2}, \ldots, k_{i} \in C^{n-i-1}, \ldots, k_{n-1} \in C^{0}, \\
& e_{1} \in C^{n-1}, \ldots, e_{i} \in C^{n-i}, \ldots, e_{n-1} \in C^{1}, e_{n} \in C^{0},
\end{aligned}
$$

but in fact $e_{n} \in C^{1}$, if we notice that $e_{n}$ can be evaluated as the direction of the orthogonal complement of span $\left\{e_{1}, \ldots, e_{n-1}\right\}$ which is $C^{1}$.

On the other hand, given $n-1$ positive continuous functions $\left\{k_{i}(s)\right\}_{1}^{n-1}$, one can recover a curve (unique up to motions) in $E^{n}$ with the curvatures $k_{i}(s)$ and the natural parameter $s$. It can be done by employment of the theorem of existence and uniqueness of the solution of a system of linear ordinary differential equations to the Frenet system (1). Since the functions $\left\{k_{i}\right\}_{1}^{n-1}$ are continuous, given an arbitrary initial orthonormal frame $\left\{e_{i}(0)\right\}_{1}^{n}$ one can recover the moving orthonormal frame $\left\{e_{i}(s)\right\}_{1}^{n}$ and it is $C^{1}$ smooth. The final radius vector of the curve $r(s)=\int_{0}^{s} e_{1}(t) d t+r(0)$ is $C^{2}$ smooth and is defined up to a choice of the initial point $r(0)$ and the initial orthonormal frame $\left\{e_{i}(0)\right\}_{1}^{n}$. Thus,

$$
\left\{k_{i}(s)\right\}_{1}^{n-1} \in C^{0}, \quad\left\{e_{i}(s)\right\}_{1}^{n} \in C^{1}, \quad r(s) \in C^{2} .
$$

Comparing (3) and (4) it is easy to see that if $k_{i}$ are only continuous, the curve is only $C^{2}$ smooth and not higher. In fact, for a curve satisfying (1) we have

$$
r \in C^{3} \Longleftrightarrow k_{1} \in C^{1}, \quad k_{2}, \ldots, k_{n-1} \in C^{0} .
$$


Indeed, $\dot{r}=e_{1}, \ddot{r}=\dot{e}_{1}=k_{1} e_{2}, \dddot{r}=\dot{k}_{1} e_{2}+k_{1} \dot{e}_{2}$. Similarly,

$$
\begin{aligned}
& r \in C^{4} \Longleftrightarrow k_{1} \in C^{2}, \quad k_{2} \in C^{1}, \quad k_{3}, \ldots, k_{n-1} \in C^{0} ; \\
& \vdots \\
& r \in C^{n} \Longleftrightarrow k_{1} \in C^{n-2}, \quad k_{2} \in C^{n-3}, \ldots, k_{n-1} \in C^{0} \text {-as in (3). }
\end{aligned}
$$

Therefore, given continuous curvatures we can recover only a $C^{2}$ smooth curve. But given a $C^{2}$ curve we can obtain neither the set of curvatures, nor the Frenet frame: we can only reach $e_{1}, e_{2}$ and $k_{1}$. Thereby the following natural question arises: to describe the exact class of curves for which the Frenet theory is satisfied (that is, the class of curves with continuous curvatures). At the moment we can only conclude that the standard analytic property of smoothness of radius vector is not flexible enough. A curve must be $C^{2}$, need not be $C^{3}$ but nevertheless has to satisfy some other conditions of goodness - evidently more geometric than smoothness of the radius vector.

This "paradox in differentiability" was noted by Hartman and Wintner in [1] for curves in $E^{3}$. They tried to clear up the situation in [1, pp.770-773] and later in [3]. In the first work the theorem of existence and uniqueness of the curve $\gamma \in E^{3}$ with prescribed continuous curvature $k_{1}$ and continuous torsion $k_{2}$ was stated (Theorem VI) but the actual class of curves was not enunciated. The second work, on the contrary, begins with the definition of the class. However, this definition is 3 -dimensional in principle and is directed rather at the treatment of rectilinear sections of a curve: a curve $r(s) \in E^{3}$ is called a Frenet curve if $r(s) \in C^{2}$ (and consequently $e_{1}=\dot{r} \in C^{1}$, $k_{1}=\left\|\dot{e}_{1}\right\|=\|\ddot{r}\| \in C^{0}$ are well-defined but $e_{2}$ is not since $k_{1}$ is allowed to be vanishing) and there exists a unit vector $e_{3}(s)$ such that $e_{3} \perp e_{1}, e_{3} \in C^{1}$, $\dot{e}_{3}$ is linearly dependant on the cross product $e_{3} \times e_{1}$.

In our opinion the following approach is more promising. Considering the Frenet system (1) we can observe that the object subjected to differentiation is not the radius vector $r(s)$ itself but only the vectors of the Frenet frame $\left\{e_{i}\right\}_{1}^{n}$. For instance, $r(s)$ can be only $C^{2}$ smooth, hence $e_{1} \in C^{1}, k_{1} \in C^{0}$. But it can occur that the nondifferentiability of $\dot{e}_{1}=k_{1} \cdot e_{2}$ is inherent in the length of the vector rather than in its direction. That is, the function $k_{1}$ is only continuous while the vector $e_{2} \in C^{1}$.

Therefore, the first thing we should take care of is the differentiability of the direction rather than the length. Denoting the direction of a vector $a$ by $\operatorname{dir}(a)=a /\|a\|$, 
the set of proper analytic conditions for $r$ is going to be

$$
\left\{\begin{aligned}
e_{1} & =\dot{r} \in C^{1} \Longleftrightarrow r \in C^{2} \\
e_{2} & =\operatorname{dir}\left(\dot{e}_{1}\right) \in C^{1} \\
e_{3} & =\operatorname{dir}\left(\dot{e}_{2}+k_{1} e_{1}\right) \in C^{1} \\
& \vdots \\
e_{i} & =\operatorname{dir}\left(\dot{e}_{i-1}+k_{i-2} e_{i-2}\right) \in C^{1} \\
& \vdots \\
e_{n-1} & =\operatorname{dir}\left(\dot{e}_{n-2}+k_{n-3} e_{n-3}\right) \in C^{1}
\end{aligned}\right.
$$

and $e_{n} \in C^{1}$ because $e_{n}=\left(\operatorname{span}\left\{e_{1}, \ldots, e_{n-1}\right\}\right)^{\perp}$. These analytic conditions are weaker than $r \in C^{n}$. They still look not very aesthetic but we can endow them with quite distinct geometric sense by introducing the following definition.

Definition 1: For a curve $r(s)$ and the point $r\left(s_{0}\right)$; the osculating 2-plane $\pi_{2}$ is the plane given by $r\left(s_{0}\right)+\operatorname{span}\left\{e_{1}, e_{2}\right\}$; the osculating 3-plane $\pi_{3}$ is the plane given by $r\left(s_{0}\right)+\operatorname{span}\left\{e_{1}, e_{2}, e_{3}\right\}$; et cetera the osculating $(n-1)$-plane $\pi_{n-1}$ is the plane given by $r\left(s_{0}\right)+\operatorname{span}\left\{e_{1}, \ldots e_{n-1}\right\}$. For the sake of completeness let us proclaim the tangent line $r\left(s_{0}\right)+\operatorname{span}\left\{e_{1}\right\}$ to be the osculating 1-plane. Hence we have the nested osculating planes $\pi_{1} \subset \pi_{2} \subset \cdots \subset$ $\pi_{n-1} \subset E^{n}$ (the flag).

Below (Part 2) we shall demonstrate the consistency of Definition 1 with the traditional one and for now we are going to complete a geometric interpretation of the system (5). The definitions of vectors $e_{i}$ in (5) look nicer than in (2), but we would still like to understand the origin of the strange items like $k_{1} e_{1}$ in the sum $\dot{e}_{2}+k_{1} e_{1}$. It turns out that they are assigned to remove the projection of $\dot{e}_{2}$ onto the osculating plane $\pi_{2}$ and leave only the projection onto $\left(\pi_{2}\right)^{\perp}$. In fact, the Frenet equation

$$
\dot{e}_{i}=-k_{i-1} e_{i-1}+k_{i} e_{i+1}
$$

is just the decomposition of $\dot{e}_{i}$ in the sum of the orthogonal projections on $\pi_{i}$ and $\left(\pi_{i}\right)^{\perp}$. Thus, for example,

$$
e_{3}=\operatorname{dir}\left(\operatorname{proj}_{\left(\pi_{2}\right)^{\perp}} \dot{e}_{2}\right) \text {. }
$$

Moreover, we can replace here the vector $e_{2}$ by an arbitrary vector from $\pi_{2}(s)$ : the derivative of any such a vector (rather, a vector field $a(s)=\alpha^{1}(s) e_{1}(s)+\alpha^{2}(s) e_{2}(s)$ ) has the projection on $\left(\pi_{2}\right)^{\perp}$ which is collinear to $e_{3}$. In other words, $e_{3}$ gives the direction of infinitesimal change (rotation) of the osculating plane $\pi_{2} ; e_{4}$ is the direction of 
infinitesimal displacement of the osculating plane $\pi_{3}$, et cetera. And if by $\dot{\pi}_{i}$ is meant the set of derivatives of all possible vector fields from $\pi_{i}$, the conditions (5) become

$$
\left\{\begin{aligned}
e_{2} & =\operatorname{dir}\left(\operatorname{proj}_{\left(\pi_{1}\right)^{\perp}}\left(\dot{\pi}_{1}\right)\right) \\
e_{3} & =\operatorname{dir}\left(\operatorname{proj}_{\left(\pi_{2}\right)^{\perp}}\left(\dot{\pi}_{2}\right)\right) \\
& \vdots \\
e_{n-1} & =\operatorname{dir}\left(\operatorname{proj}_{\left(\pi_{n-2}\right)^{\perp}}\left(\dot{\pi}_{n-2}\right)\right) .
\end{aligned}\right.
$$

Now a geometric interpretation of the conditions (5) can be presented:

$$
\begin{gathered}
e_{1}(s) \in C^{1} \Longleftrightarrow \exists ! \text { tangent (osculating 1-plane) and it is } C^{1} \text { smooth; } \\
e_{2}(s) \in C^{1} \Longleftrightarrow \exists ! \text { osculating 2-plane and it is } C^{1} \text { smooth; } \\
e_{3}(s) \in C^{1} \Longleftrightarrow \exists ! \text { osculating 3-plane and it is } C^{1} \text { smooth; } \\
\vdots \\
e_{n-1}(s) \in C^{1} \Longleftrightarrow \exists ! \text { osculating }(n-1) \text {-plane and it is } C^{1} \text { smooth. }
\end{gathered}
$$

REMARK. Further relaxation of smoothness conditions, say to $e_{i} \in C^{0,1}$ (the Lipschitz condition of order 1 ) instead of $e_{i} \in C^{1}$ appears impossible since in this case the direction of the vector $e_{i+1}$ is not well-defined.

Finally we can state two equivalent theorems: an analytic Theorem 1 and a geometric Theorem 2.

THEOREM 1. A curve $\gamma \subset E^{n}$ possesses continuous curvatures if and only if its radius vector $r(s)$ satisfies the system (5) with the set $\left\{e_{i}\right\}_{1}^{n}$ defined by (2).

Theorem 2. A curve $\gamma \subset E^{n}$ possesses continuous curvatures if and only if at every point the flag of osculating planes $\pi_{1} \subset \pi_{2} \subset \cdots \subset \pi_{n-1} \subset E^{n}$ exists and is unique and this flag is $C^{1}$ smooth.

Therefore, the class of curves, defined by the conditions $(2)+(5)$ seems quite rich in content and deserves the name of the class of Frenet curves.

Note, such a definition is quite consistent with the definition by Wintner [3] cited earlier. Moreover, in our terminology that long definition becomes perfectly transparent: a curve is called a Frenet curve if it possesses a $C^{1}$ smooth Frenet frame $\left\{e_{i}\right\}_{1}^{3}$. Therefore, Wintner's definition is different from ours only by dropping the condition of uniqueness of the frame (rather, there is freedom for $e_{2}$ or $e_{3}$, while the tangent vector $e_{1}$ is well defined) - that should be expected when we want to consider the curves with rectilinear sections. 


\section{Osculating planes}

Traditionally, the osculating $k$-plane of a curve $r(s)$ at the point $r\left(s_{0}\right)$ is defined as the plane through $r\left(s_{0}\right)$ spanned by $r^{\prime}\left(s_{0}\right), \ldots, r^{(k)}\left(s_{0}\right)$. We have dropped the condition of multiple differentiability of $r(s)$ but still can define what is required. And even in two ways.

Definition 2: The osculating $k$-plane of $r(s)$ at $r\left(s_{0}\right)$ is the $k$-plane $\pi_{k}$ which is closest to the curve $r(s)$ among all $k$-planes passing through $r\left(s_{0}\right)$. The closeness between a curve and a plane is measured in terms of the order of osculation-the order of smallness of the distance from $r(s)$ to the plane: $\operatorname{dist}\left(r\left(s_{0}+\Delta s\right), \pi_{k}\right)$. For the osculating $k$-plane the order of osculation is $k+1$, that is, $\operatorname{dist}\left(r, \pi_{k}\right) \sim(\Delta s)^{k+1}$.

Definition 3: The osculating k-plane of $r(s)$ at $r\left(s_{0}\right)$ is the limit position of $k$-planes passing through $k+1$ distinct points $r\left(s_{1}\right), \ldots, r\left(s_{k+1}\right)$ as $s_{i} \longrightarrow s_{0}$.

We restrict ourselves to the demonstration of equivalence of Definition 1 and Definition 2 for curves from the class. However, one can show the equivalence of them to Definition 3 (see [2, pp.32-34]).

THEOREM 3. For a curve $r(s) \subset E^{n}$ possessing continuous curvatures, the plane $\pi_{k}: r\left(s_{0}\right)+\operatorname{span}\left\{e_{1}\left(s_{0}\right), \ldots, e_{k}\left(s_{0}\right)\right\}$ (with $\left\{e_{i}\right\}$ given by $(2)$ ) is the closest to the curve at $r\left(s_{0}\right)$ among all $k$-planes through $r\left(s_{0}\right)$. The order of osculation of $\pi_{k}$ and $r(s)$ at $r\left(s_{0}\right)$ is $k+1$ and for any other $k$-plane the order is smaller.

Proof: Without loss of generallity we assume $s_{0}=0, r(0)=0$. Let us decompose the curve $r(s)$ by the basis $\left\{e_{i}(0)\right\}_{1}^{n}$ :

$$
r(s)=\alpha^{i}(s) e_{i}(0)
$$

We intend to show

$$
\left\{\begin{aligned}
\alpha^{1} & =s+o(s) \\
\alpha^{2} & =\frac{1}{2} k_{1}(0) s^{2}+o\left(s^{2}\right) \\
\alpha^{3} & =\frac{1}{6} k_{1}(0) k_{2}(0) s^{3}+o\left(s^{3}\right) \\
& \vdots \\
\alpha^{j} & =\frac{1}{j !} k_{1}(0) \cdot \ldots \cdot k_{j-1}(0) s^{j}+o\left(s^{j}\right) \\
& \vdots \\
\alpha^{n} & =\frac{1}{n !} k_{1}(0) \cdot \ldots \cdot k_{n-1}(0) s^{n}+o\left(s^{n}\right)
\end{aligned}\right.
$$


It is not difficult to see that this system implies the conclusion of Theorem 3.

If $r(s)$ were $C^{n}$ the system (9) could be rewritten as follows

$$
\left\{\begin{array}{l}
\left(\alpha^{j}\right)^{(i)}(0)=\left\langle r^{(i)}(0), e_{j}(0)\right\rangle=0 \quad(i<j) \\
\left(\alpha^{j}\right)^{(j)}(0)=\left\langle r^{(j)}(0), e_{j}(0)\right\rangle=k_{1}(0) \cdot \ldots \cdot k_{j-1}(0),
\end{array}\right.
$$

where $j$ runs through the values 1 to $n$. The same condition but with fixed $i$ instead of $j$ has the form

$$
\left\{\begin{array}{l}
\left\langle r^{(i)}(0), e_{j}(0)\right\rangle=0 \quad(j>i) \\
\left\langle r^{(i)}(0), e_{i}(0)\right\rangle=k_{1}(0) \cdot \ldots \cdot k_{i-1}(0)
\end{array}\right.
$$

where $i$ runs 1 to $n$. The last system, as can be easily seen, is implied by

$$
\begin{aligned}
r^{(i)} & =\left(e_{1}\right)^{(i-1)}=\left(k_{1} e_{2}\right)^{(i-2)}=\left(A_{1} e_{1}+A_{2} e_{2}+k_{1} k_{2} e_{3}\right)^{(i-3)} \\
& =B_{1} e_{1}+B_{2} e_{2}+\cdots+B_{i-1} e_{i-1}+k_{1} k_{2} \ldots k_{i-1} e_{i}
\end{aligned}
$$

(here $A_{i}, B_{i}$ are some functions depending on $k_{j}$ and their derivatives).

Since $r(s)$ is not $C^{n}$ we have to change somehow the techniques of proof of the formulae (9). From $r \in C^{2}$ we can still extract

$$
\begin{array}{lll}
r(0)=0 & \Longleftrightarrow & \alpha^{i}(0)=0 \\
\dot{r}(0)=e_{1}(0) & \Longleftrightarrow & \dot{\alpha}^{i}(0)=\delta_{1}^{i} \\
\ddot{r}(0)=k_{1}(0) e_{2}(0) & \Longleftrightarrow & \ddot{\alpha}^{i}(0)=k_{1}(0) \delta_{2}^{i},
\end{array}
$$

which gives

$$
\left\{\begin{array}{l}
\alpha^{1}=s+o\left(s^{2}\right) \\
\alpha^{2}=\frac{1}{2} k_{1}(0) s^{2}+o\left(s^{2}\right) \\
\alpha^{i}=o\left(s^{2}\right) \quad \text { for } i>2 .
\end{array}\right.
$$

The next condition $e_{2}(s) \in C^{1}$ allows us to evaluate the coefficients of $s^{3}$ for the system (9). We intend to show that

$$
\left\{\begin{array}{l}
\alpha^{3}=\frac{1}{6} k_{1}(0) k_{2}(0) s^{3}+o\left(s^{3}\right) \\
\alpha^{i}=o\left(s^{3}\right) \quad \text { for } i>3 .
\end{array}\right.
$$

Indeed, from $e_{2}(s) \stackrel{(2)}{=} \ddot{r}(s) / k_{1}(s) \in C^{1}$ we have

$$
\begin{aligned}
-k_{1}(0) e_{1}(0)+k_{2}(0) e_{3}(0) \stackrel{(1)}{=} \dot{e}_{2}(0) \stackrel{\text { def }}{=} \lim _{s \rightarrow 0} \frac{1}{s}\left(\frac{\ddot{r}(s)}{k_{1}(s)}-\frac{\ddot{r}(0)}{k_{1}(0)}\right) \\
\stackrel{(8)}{=} \lim _{s \rightarrow 0} \frac{1}{s}\left(\frac{\ddot{\alpha}^{i}(s)}{k_{1}(s)} e_{i}(0)-e_{2}(0)\right)=e_{i}(0) \lim _{s \rightarrow 0} \frac{1}{s}\left(\frac{\ddot{\alpha}^{i}(s)}{k_{1}(s)}-\delta_{i}^{2}\right) .
\end{aligned}
$$


The coefficients of $e_{3}(0)$ give

$$
k_{2}(0)=\lim _{s \rightarrow 0} \frac{\ddot{\alpha}^{3}(s)}{k_{1}(s) \cdot s}=\frac{1}{k_{1}(0)} \lim _{s \rightarrow 0} \frac{\ddot{\alpha}^{3}(s)}{s} .
$$

Using (10) we have the first equation of (11). In the same way by considering coefficients of $e_{i}(0), i>3$ we have the rest of the system (11). Combining (10) and (11) one has

$$
\left\{\begin{array}{l}
\alpha^{1}=s+o\left(s^{2}\right) \\
\alpha^{2}=\frac{1}{2} k_{1}(0) s^{2}+o\left(s^{2}\right) \\
\alpha^{3}=\frac{1}{6} k_{1}(0) k_{2}(0) s^{3}+o\left(s^{3}\right) \\
\alpha^{i}=o\left(s^{3}\right) \quad \text { for } i>3 .
\end{array}\right.
$$

In the next step we shall use the condition $e_{3}(s) \in C^{1}$ in order to have

$$
\left\{\begin{array}{l}
\alpha^{4}=\frac{1}{4 !} k_{1}(0) k_{2}(0) k_{3}(0) s^{4}+o\left(s^{4}\right) \\
\alpha^{i}=o\left(s^{4}\right) \quad \text { for } i>4 .
\end{array}\right.
$$

So, $e_{3}(s) \stackrel{(2)}{=}\left(1 / k_{2}\right)\left(\left(\ddot{r} / k_{1}\right)^{\prime}+k_{1} \dot{r}\right) \in C^{1}$. Evaluating the derivative of $e_{3}$ at 0 gives

$$
\begin{aligned}
-k_{2}(0) & e_{2}(0)+k_{3}(0) e_{4}(0) \stackrel{(1)}{=} \dot{e}_{3}(0) \\
& \stackrel{\text { def }}{=} \lim _{s \rightarrow 0} \frac{1}{s}\left[\frac{1}{k_{2}(s)}\left(\left(\frac{\ddot{r}}{k_{1}}\right)^{\prime}+k_{1} \dot{r}\right)(s)-\frac{1}{k_{2}(0)}\left(\left(\frac{\ddot{r}}{k_{1}}\right)^{\prime}+k_{1} \dot{r}\right)(0)\right] \\
& \stackrel{(8)}{=} \lim _{s \rightarrow 0} \frac{1}{s}\left[\frac{1}{k_{2}(s)}\left(\left(\frac{\ddot{\alpha}^{i}}{k_{1}}\right)^{\prime}+k_{1} \dot{\alpha}^{i}\right)(s)-\frac{1}{k_{2}(0)}\left(\left(\frac{\ddot{\alpha}^{i}}{k_{1}}\right)^{\prime}+k_{1} \dot{\alpha}^{i}\right)(0)\right] e_{i}(0) .
\end{aligned}
$$

The coefficients of $e_{4}(0)$ :

$$
\begin{aligned}
k_{3}(0)= & \lim _{s \rightarrow 0} \frac{1}{s}\left[\frac{1}{k_{2}(s)}\left(\left(\frac{\ddot{\alpha}^{4}}{k_{1}}\right)^{\prime}+k_{1} \dot{\alpha}^{4}\right)(s)-\frac{1}{k_{2}(0)}\left(\left(\frac{\ddot{\alpha}^{4}}{k_{1}}\right)^{\prime}+k_{1} \dot{\alpha}^{4}\right)(0)\right] \\
= & \frac{1}{k_{2}(0)} \lim _{s \rightarrow 0} \frac{1}{s}\left(\frac{\ddot{\alpha}^{4}}{k_{1}}\right)^{\prime}(s)+\frac{k_{1}(0)}{k_{2}(0)} \lim _{s \rightarrow 0} \frac{\dot{\alpha}^{4}(s)}{s} \\
& \quad-\frac{1}{k_{2}(0)} \lim _{s \rightarrow 0} \frac{1}{s}\left(\frac{\ddot{\alpha}^{4}}{k_{1}}\right)^{\prime}(0)-\frac{k_{1}(0)}{k_{2}(0)} \lim _{s \rightarrow 0} \frac{\dot{\alpha}^{4}(0)}{s} .
\end{aligned}
$$

Using $\alpha^{4}(s)=o\left(s^{3}\right)$ it is clearly seen that the second and the fourth limits vanish. The third limit vanishes too:

$$
\begin{aligned}
& \lim _{s \rightarrow 0} \frac{1}{s}\left(\frac{\ddot{\alpha}^{4}}{k_{1}}\right)^{\prime}(0) \stackrel{\text { def }}{=} \lim _{s \rightarrow 0} \frac{1}{s} \lim _{t \rightarrow 0} \frac{1}{t}\left[\frac{\ddot{\alpha}^{4}(t)}{k_{1}(t)}-\frac{\ddot{\alpha}^{4}(0)}{k_{1}(0)}\right] \stackrel{(12)}{=} \frac{1}{k_{1}(0)} \lim _{s \rightarrow 0} \frac{1}{s}\left[\lim _{t \rightarrow 0} \frac{\ddot{\alpha}^{4}(t)}{t}\right] \\
& \stackrel{(12)}{=} \frac{1}{k_{1}(0)} \lim _{s \rightarrow 0}\left(\frac{1}{s} \cdot 0\right)=0 \text {. }
\end{aligned}
$$


It remains to consider the first limit. As $\alpha^{4}(s)=o\left(s^{3}\right)$ let $\alpha^{4}(s)=(1 / 4 !) C s^{4}+o\left(s^{4}\right)$. Then the first limit is

$$
\begin{aligned}
k_{3}(0) & =\frac{1}{k_{2}(0)} \lim _{s \rightarrow 0} \frac{1}{s} \lim _{t \rightarrow 0} \frac{1}{t}\left[\frac{\ddot{\alpha}^{4}(s+t)}{k_{1}(s+t)}-\frac{\ddot{\alpha}^{4}(s)}{k_{1}(s)}\right] \\
& =\frac{1}{k_{1}(0) k_{2}(0)} \lim _{s \rightarrow 0} \frac{1}{s} \lim _{t \rightarrow 0} \frac{1}{t} \frac{C}{2}\left[(s+t)^{2}+o\left((s+t)^{2}\right)-s^{2}-o\left(s^{2}\right)\right] \\
& =\frac{C}{k_{1}(0) k_{2}(0)} .
\end{aligned}
$$

So, $C=k_{1}(0) k_{2}(0) k_{3}(0)$ as was claimed in (13). The same reasoning for the coefficients of $e_{i}(i>4)$ gives the rest of the system (13).

Similarly, the condition $e_{4}(s) \in C^{1}$ provides the coefficients of $s^{5}$ in (9), et cetera.

\section{REFERENCES}

[1] $\mathrm{Ph}$. Hartman and $\mathrm{A}$. Wintner, 'On the fundamental equations of differential geometry', Amer. J. Math. 72 (1950), 757-774.

[2] M. Spivak, A comprehensive introduction to differential geometry II (Publish or Perish, Inc., Berkeley, 1979).

[3] A. Wintner, 'On Frenet's equations', Amer. J. Math. 78 (1956), 349-356.

Department of Mathematics The University of Melbourne Parkville Vic 3052

Australia

e-mail: vit@mundoe.maths.mu.oz.au 